GLASNIK MATEMATIČKI

Vol. 38(58)(2003), $111-120$

\title{
A NOTE ON QUASI-ISOMETRIES II
}

\author{
S.M. PATEL \\ Sardar Patel University, India
}

\begin{abstract}
An operator $A$ on a complex Hilbert space $H$ is called a quasi-isometry if $A^{*^{2}} A^{2}=A^{*} A$. In the present article, some structural properties of quasi-isometries are established with the help of operator matrix representation.
\end{abstract}

\section{INTRODUCTION}

A bounded linear transformation of a complex Hilbert space $H$ into itself is called an operator on $H$. In [6], we have introduced the concept of a quasi-isometry which is defined as an operator $A$ for which $A^{*^{2}} A^{2}=A^{*} A$ or equivalently, $\left\|A^{2} x\right\|=\|A x\|$ for all $x \in H$. Obviously the class of quasiisometries is a simple extension of isometries. The purpose of the present exposition is to explore some properties of quasi-isometries by exploiting the special kind of operator matrix representation associated with such operators. In the course of our investigation, we find some properties of isometries, which are retained by quasi-isometries. However, there are other ones, which are shown to be false for quasi-isometries.

\section{Notations AND TERMinOlogy}

We use the notations $N(A)$ and $R(A)$ respectively for the null space and the range of an operator $A$. The symbol $\bar{F}$ will be used for the closure of a set $F$. We write $\sigma(A), \pi_{0}(A), \pi_{00}(A), w(A)$ and $W(A)$ respectively, for the spectrum, the point spectrum, the set of eigenvalues of finite multiplicity, the Weyl spectrum and the numerical range of $A$. Let $r(A)$ and $|W(A)|$ denote the spectral radius and the numerical radius of $A$. For an operator $A$, if $w(A)=\sigma(A) \backslash \pi_{00}(A)$, then we say that the Weyl's theorem holds for $A$.

2000 Mathematics Subject Classification. 47A62, 47A63, 47B20.

Key words and phrases. Hilbert space, isometry, spectrum, operator matrix. 


\section{RESULTS}

We begin with the following result that is the heart of our entire exposition.

THEOREM 3.1. If an operator $A$ is a quasi-isometry, then it has an operator matrix representation given by

$$
A=\left[\begin{array}{ll}
T & S \\
0 & 0
\end{array}\right],
$$

where $T$ is an isometry and $S$ is a bounded linear transformation.

Proof. Let $H=\overline{R(A)} \oplus N\left(A^{*}\right)$ and $P$, the projection on $\overline{R(A)}$. Then $A$ has the operator matrix representation

$$
A=\left[\begin{array}{ll}
T & S \\
0 & 0
\end{array}\right],
$$

where $T=A / \overline{R(A)}$ and $S=P A / N\left(A^{*}\right)$. If $y=A x$, then $\|T y\|=\|A y\|=$ $\left\|A^{2} x\right\|=\|A x\|=\|y\|$ and so $\|T y\|=\|y\|$ for $y \in \overline{R(A)}$. This means that the operator $T$ is an isometry.

REMARK 3.2. The converse of preceding theorem is also true. In fact, if $T$ is an isometry on $H$ and if $S$ is a bounded linear transformation from a Hilbert space $H$ to another Hilbert space $K$, then it is easy to show that the operator matrix is a quasi-isometry acting on $H \oplus K$.

In[6, Theorem 2], we proved that the following statements are equivalent for a quasi-isometry $A$.

(i) $\|A\|=1$.

(ii) $A$ is hyponormal.

In the next result we use the matrix representation to derive yet another statement which is equivalent to both (i) and (ii).

TheOREM 3.3. Let

$$
A=\left[\begin{array}{ll}
T & S \\
0 & 0
\end{array}\right]
$$

where $T$ is an isometry. Then $\|A\|=1$ if and only if (a) $S^{*} T=0$, and (b) $\|S\| \leq 1$.

Proof. Since

$$
A A^{*}=\left[\begin{array}{cc}
T T^{*}+S S^{*} & 0 \\
0 & 0
\end{array}\right],
$$

$\|A\|^{2}=\left\|T T^{*}+S S^{*}\right\|$. Suppose $\|A\|=1$. Then $T T^{*}+S S^{*} \leq I$ and so $\|S\| \leq 1$. Also

$$
\left\|I+T^{*} S S^{*} T\right\|=\left\|T^{*}\left(T T^{*}+S S^{*}\right) T\right\| \leq\left\|T T^{*}+S S^{*}\right\|=1
$$


Suppose $\alpha \in \sigma\left(I+T^{*} S S^{*} T\right)$. Then $\alpha-1 \in \sigma\left(T^{*} S S^{*} T\right)$; thus $\alpha \geq 1$ and hence by (3.1), $\alpha=1$. As a consequence of this, we find $T^{*} S S^{*} T=0$ or $S^{*} T=0$; thus the assertions (a) and (b) hold. Assume the converse. Observe that

$$
A^{*} A=\left[\begin{array}{ll}
T^{*} T & T^{*} S \\
S^{*} T & S^{*} S
\end{array}\right]=\left[\begin{array}{ll}
I & 0 \\
0 & S^{*} S
\end{array}\right]
$$

gives $\|A\|=\max \{1,\|S\|\}$. Since $\|S\| \leq 1,\|A\|=1$.

It is obvious that an idempotent operator is a quasi-isometry with spectrum consisting of at the most two points 0 and 1 . In the next result we establish the converse. In the proof of the result and the following one, we shall use the following observation: given operators $T$ and $S$ if

$$
A=\left[\begin{array}{ll}
T & S \\
0 & 0
\end{array}\right]
$$

then $\sigma(A)$ contains all non-zero elements of $\sigma(T)$.

Theorem 3.4. A quasi-isometry $A$ is idempotent provided $\sigma(A) \subseteq\{0,1\}$.

Proof. Let

$$
A=\left[\begin{array}{ll}
T & S \\
0 & 0
\end{array}\right]
$$

as in Theorem 3.1. The observation stated just before the present theorem along with the hypothesis on $\sigma(A)$ shows that $\sigma(T)$ consists of 1 only. Since $T$ is an isometry, it follows that $T=I$. This proves the result.

A further relaxation in the hypothesis of the preceding theorem gives the following result.

TheOREM 3.5. If $A$ is a quasi-isometry with real spectrum, then $A^{3}=A$.

Proof. We suppose that $A$ has the matrix representation given in Theorem 3.1. By our hypothesis, we find that $\sigma(T)$ is real. Since $T$ is a hyponormal operator, the convex hull of $\sigma(T)$ equals $\overline{W(T)}$ [8]. Therefore $\overline{W(T)}$ is real or $T^{*}=T$. Hence $T^{2}=I$. Now it is easy to check the relation $A^{3}=A$.

THEOREM 3.6. Let

$$
A=\left[\begin{array}{ll}
T & S \\
0 & 0
\end{array}\right]
$$

be a quasi-isometry. If $A^{*}$ is also a quasi-isometry, then $T$ is unitary and $\left(A^{*} A\right)^{2} \geq\left(A^{*} A\right)$.

Proof. First we assert that $T$ is unitary. It is enough to show that $T^{*}$ is injective. Suppose $T^{*} x=0$. Since $A^{2} A^{* 2}=A A^{*}$, a computation shows that

$$
T^{2} T^{* 2}+T S S^{*} T^{*}=T T^{*}+S S^{*}
$$


From this it is immediate that $S S^{*} x=0$ or $S^{*} x=0$. Now premultiplying and postmultiplying (3.2) by $T^{*}$ and $T$ respectively, we get

$$
T T^{*}+S S^{*}=I+T^{*} S S^{*} T
$$

Since $T^{*} x=0=S^{*} x$, we deduce $x+T^{*} S S^{*} T x=0$ from $(3.3)$ or $0=$ $\|x\|^{2}+\left\|S^{*} T x\right\|^{2}$. Hence $x=0$ which shows the injectivity of $T$. Now we verify the remaining assertion. We denote the operator matrix

$$
\left[\begin{array}{ll}
I & 0 \\
0 & 0
\end{array}\right]
$$

by $P$. The fact that $T$ is unitary yields

$$
A A^{*}-A P A^{*}=\left[\begin{array}{cc}
S S^{*} & 0 \\
0 & 0
\end{array}\right]
$$

and

$$
A P A^{*} A=A
$$

Postmultiplying equation (3.4) by $A$ and using equation (3.5), we get

$$
A A^{*} A-A=\left[\begin{array}{cc}
S S^{*} & 0 \\
0 & 0
\end{array}\right]\left[\begin{array}{ll}
T & S \\
0 & 0
\end{array}\right]
$$

which in turn gives

$$
\left(A^{*} A\right)^{2}-A^{*} A=A^{*}\left[\begin{array}{cc}
S S^{*} & 0 \\
0 & 0
\end{array}\right] A \geq 0
$$

Although an isometry is left invertible, the corresponding result for quasiisometries is not true (Counter Example: an idempotent operator which not the identity operator). This motivates us to pay special attention to left invertible quasi-isometries.

TheOREm 3.7. A left invertible quasi-isometry is similar to an isometry.

Proof. Let $A$ be a left invertible quasi-isometry. Define a new inner product on $H$ by $<x, y>_{o}=<A x, A y>$. Clearly $\|x\|_{o}=\|A x\| \leq\|A\|\|x\|$. Since $A$ is bounded below,

$$
M\|x\| \leq\|x\|_{o} \leq\|A\|\|x\|
$$

for some $M>0$. This shows that $H$ is also a Hilbert space with respect to new inner product. Denote this new Hilbert space by $H_{o}$. Let $I$ from $H$ to $H_{o}$ be the identity transformation. The continuity of $I$ follows from (3.6). Let $A_{o}=I A I^{-1}$. Then $A_{0}$ is an isometry. This proves the result.

REMARK 3.8. The preceding theorem insipires us to examine the existence of a stronger possibility for a left invertible quasi-isometry to be an isometry. However, the following example ruled out this possibility. 
ExAmple 3.9. Define operators $T$ and $S$ on a Hilbert space $H=l^{2}$ by

$$
T\left(x_{1}, x_{2}, x_{3}, \ldots\right)=\left(0, x_{1}, 0, x_{2}, . .\right)
$$

and

$$
S\left(x_{1}, x_{2}, x_{3}, \ldots\right)=\left(2 x_{1}, 0, x_{2}, 0, x_{3}, 0, \ldots\right) .
$$

Then it is not difficult to show that

(i) $T$ is an isometry,

(ii) $S^{*} T=0$,

(iii) $\|S x\| \geq\|x\|$ for all $\mathrm{x}$ in $H$,

(iv) $S$ is not an isometry as $\left\|S e_{1}\right\|=2$ for $e_{1}=(1,0,0, \ldots)$.

We assert that the operator

$$
A=\left[\begin{array}{ll}
T & S \\
0 & 0
\end{array}\right]
$$

is bounded below. If not, then there is a sequence of unit vectors $z_{n}=\left(x_{n}, y_{n}\right)$ in $H \oplus H$ such that $A z_{n} \rightarrow 0$ or $T x_{n}+S y_{n} \rightarrow 0$. Because $T$ is an isometry and $S^{*} T=0$, we find $x_{n} \rightarrow 0$ and hence $S y_{n} \rightarrow 0$. By (iii), $y_{n} \rightarrow 0$. Thus $A$ is a quasi-isometry that is bounded below. However, if we let $z=(1 \sqrt{ } 2)\left(e_{1}, e_{1}\right)$, then $\langle A z, z>=3 / 2$, resulting $\|A\|>1$. Consequently $A$ fails to be an isometry.

The following theorem appeared in [6] is proved here with an alternate argument that uses the operator matrix representation.

THEOREM 3.10. An isolated point in the spectrum of a quasi-isometry is an eigen-value.

ProOF. Let

$$
A=\left[\begin{array}{ll}
T & S \\
0 & 0
\end{array}\right]
$$

be a quasi-isometry. Let $\lambda$ be an isolated point in $\sigma(A)$. Suppose $\lambda$ is zero. If $0 \in \sigma(T)$, then 0 is an eigen-value of $T$ because $T$ is hyponormal and we know that an isolated point in the spectrum of a hyponormal operator is an eigenvalue [7]. But this will contradicts the injectivity of $T$. Therefore 0 cannot be in $\sigma(T)$ and so $T$ must be unitary. Fix a non-zero vector y in $H$. Then there is a non-zero vector $x$ such that $S y=-T x$ or $A(x, y)=0$. This shows that 0 is an eigen-value of $A$. Next assume that $\lambda$ is non-zero. Clearly it is an isolated point of $\sigma(T)$ and so an eigen-value of $T$. Choose a non-zero vector $x$ such that $(T-\lambda I) x=0$. Clearly $A(x, 0)=\lambda(x, 0)$. This completes the proof.

Theorem 3.11. Let

$$
A=\left[\begin{array}{ll}
T & S \\
0 & 0
\end{array}\right]
$$


be a quasi-isometry with $S^{*} T=0$. Then $R(S)$ is closed if and only if $R(A)$ is closed.

Proof. Suppose $R(S)$ is closed. Let $\left\{\left(x_{n}, y_{n}\right)\right\}$ be the sequence of vectors such that $A\left(x_{n}, y_{n}\right) \rightarrow(x, y)$. Clearly $T x_{n}+S y_{n} \rightarrow x$ and $y=0$. Since $T$ is an isometry, it follows that $x_{n}+T^{*} S y_{n} \rightarrow T^{*} x$ and so $x_{n} \rightarrow T^{*} x$ as $T^{*} S=0$. This, in turn, gives $S y_{n} \rightarrow x-T T^{*} x$ and $T x_{n} \rightarrow T T^{*} x$. Since $R(S)$ is closed, $x-T T^{*} x=S u$ for some $\mathrm{u}$ in $H$. Thus $x=T T^{*} x+S u$ which shows that $(x, y) \in R(A)$.

On the other hand if $R(A)$ is closed, then $(y, 0) \in R(A)$ whenever $S y_{n} \rightarrow$ $y$. This gives $y=T x+S z$ for some vectors $x$ and $z$. Since $T^{*} S=0, T^{*} y=0$ and hence $0=x+T^{*} S z=x$ giving $y=S z$; thus $R(S)$ is closed.

REMARK 3.12. As an application of the preceding theorem, we construct a quasi-isometry to show that unlike the range of an isometry, the range of a quasi-isometry need not be closed

Example 3.13. Define operators $T$ and $S$ on $\ell^{2}$ by

$$
T\left(x_{1}, x_{2}, x_{3}, \ldots\right)=\left(0, x_{1}, 0, x_{2}, 0, \ldots\right)
$$

and

$$
S\left(x_{1}, x_{2}, x_{3}, \ldots\right)=\left(x_{1}, 0, x_{2} / 2,0, x_{3} / 3,0, \ldots\right) .
$$

Since $\operatorname{dim} R(S)=\infty$ and $\mathrm{S}$ is a compact operator, $R(S)$ cannot be closed. Also it is easy to verify that $S^{*} T=0$. In view of the above result $R(A)$ fails to be closed.

It is well known that Weyl's theorem holds for hyponormal operators [2] and in particular. Here we find that this important property of hyponormal operators (and in particular of quasi-isometries) is also shared by quasiisometries.

In the sequel, $A$ will denote a quasi-isometry with the matrix representation as obtained in Theorem 3.1. In order to prove Weyl's theorem for $A$, we shall need some lemmas. We assume that $A$ is a non-unitary operator.

Lemma 3.14. For a non-zero complex number $z, R(A-z I)$ is closed if and only if $R(T-z I)$ is closed.

Proof. Suppose $R(A-z I)$ is closed and $(T-z I) x_{n} \rightarrow x$. Then $(A-$ $z I)\left(x_{n}, 0\right) \rightarrow(x, 0)$. Since $R(A-z I)$ is closed, there exist vectors $\mathrm{u}$ and $\mathrm{v}$ such that $(x, 0)=(A-z I)(u, v)$ or $x=(T-z I) u+S v$ and $0=z v$. Because $z$ is non-zero, we find $v=0$ resulting in $x=(T-z I) u$. This proves that $R(T-z I)$ is closed.

Conversely assume that $R(T-z I)$ is closed. Let $\left\{\left(x_{n}, y_{n}\right)\right\}$ be a sequence of vectors for which $(A-z I)\left(x_{n}, y_{n}\right) \rightarrow(x, y)$ or $(T-z I) x_{n}+S y_{n} \rightarrow x$ and $-z y_{n} \rightarrow y$. Since $z$ is non-zero, $y_{n} \rightarrow-(1 / z) y$ and hence $(T-z I) x_{n} \rightarrow$ $x+(1 / z) S y$. Since $R(T-z I)$ is closed, we find a vector u such that $x+$ 
$(1 / z) S y=(T-z I) u$. It is clear that $(A-z I)(u,-y / z)=(x, y)$. This shows that $R(A-z I)$ is closed.

Lemma 3.15. For a non-zero complex number $z$,

(i) $\operatorname{dim} N(A-z I)=\operatorname{dim} N(T-z I)$.

(ii) $\operatorname{dim} N\left(A^{*}-z^{*} I\right)=\operatorname{dim} N\left(T^{*}-z^{*} I\right)$.

Proof. (i) is obvious in view of the relation $N(A-z I)=N(T-z I) \cup\{0\}$. Now we prove (ii). Note that $(x, y) \in N\left(A^{*}-z^{*} I\right)$ if and only if $x \in N\left(T^{*}-\right.$ $\left.z^{*} I\right)$ and $y=S^{*} x / z^{*}$.

Suppose $\left(x_{1}, y_{1}\right), \ldots,\left(x_{m}, y_{m}\right)$ are linearly independent vectors in $N\left(A^{*}-\right.$ $\left.z^{*} I\right)$. Then $x_{i} \in N\left(T^{*}-z^{*} I\right)$ and $y_{i}=S^{*} x_{i} / z^{*}$ for $i=1,2,3, \ldots$ If $\alpha_{1} x_{1}+$ $\alpha_{2} x_{2}+\alpha_{3} x_{3}+\cdots+\alpha_{m} x_{m}=0$, then $\alpha_{1} y_{1}+\alpha_{2} y_{2}+\alpha_{3} y_{3}+\cdots+\alpha_{m} y_{m}=0$ and so $\alpha_{1}\left(x_{1}, y_{1}\right)+\alpha_{2}\left(x_{2}, y_{2}\right)+\alpha_{3}\left(x_{3}, y_{3}\right)+\cdots+\alpha_{m}\left(x_{m}, y_{m}\right)=0$. Since vectors $\left(x_{i}, y_{i}\right)(i=1,2,3, \ldots, m)$ are linearly independent, it follows that $\alpha_{i}=0$. This means that the vectors $x_{1}, x_{2}, \ldots, x_{m}$ are linearly independent. Hence

$$
\operatorname{dim} N\left(A^{*}-z^{*} I\right) \leq \operatorname{dim} N\left(T^{*}-z^{*} I\right) .
$$

Next we obtain the reverse inequality. Let $x_{1}, x_{2}, \ldots, x_{n}$ be linearly independent vectors in $N\left(T^{*}-z^{*} I\right)$. Set $y_{i}=S^{*} x_{i} / z^{*}$. Then vectors $\left(x_{1}, y_{1}\right), \ldots,\left(x_{n}, y_{n}\right)$ belong to $N\left(A^{*}-z^{*} I\right)$. The linear independence of these vectors follows from that of $x_{1}, x_{2}, \ldots, x_{n}$. Consequently

$$
\operatorname{dim} N\left(A^{*}-z^{*} I\right) \geq \operatorname{dim} N\left(T^{*}-z^{*} I\right) .
$$

From (3.7) and (3.8), our second assertion follows.

LEMMA 3.16. $\sigma(A) \backslash\{0\}=\left(w(A) \cup \pi_{o o}(A)\right) \backslash\{0\}$.

Proof. By Lemma 3.14 and Lemma 3.15, we find

$$
w(A) \backslash\{0\}=w(T) \backslash\{0\}
$$

and

$$
\pi_{o o}(A) \backslash\{0\}=\pi_{o o}(T) \backslash\{0\} .
$$

Since $T$ is an isometry, $\sigma(T)=w(T) \cup \pi_{o o}(T)$. Therefore $\sigma(A) \backslash\{0\}=$ $\sigma(T) \backslash\{0\}=\left(w(T) \cup \pi_{o o}(T)\right) \backslash\{0\}=(w(T) \backslash\{0\}) \cup\left(\pi_{o o}(T) \backslash\{0\}\right)=(w(A) \cup$ $\left.\pi_{\text {oo }}(A)\right) \backslash\{0\}$.

LEMMA 3.17. $0 \in w(A) \cup \pi_{o o}(A)$.

Proof. Suppose $0 \notin w(A) \cup \pi_{o o}(A)$. Then either 0 is not an isolated point of $\sigma(A)$ or is an eigenvalue of $A$ with infinite multiplicity. In the latter case, $0 \in w(A)$ which contradicts the assumption $0 \notin w(A) \cup \pi_{o o}(A)$. Therefore the only possibility remains with us is that 0 is a limit point of $\sigma(A)$. Select a sequence $\left\{z_{n}\right\}$ of non-zero distinct points from $\sigma(A)$ converging to 0 . In view of Lemma 3.15, each $z_{n}$ lies in $w(A) \cup \pi_{o o}(A)$. Because of our assumption that $0 \notin w(A) \cup \pi_{o o}(A), w(A)$ can contain at the most finitely many $z_{n}^{\prime} s$. 
Therefore there exists a positive integer $\mathrm{k}$ such that $z_{n} \in \pi_{o o}(A)$ for $n \geq k$. In particular, $z_{n} \in \partial \sigma(A)$ for $n \geq k$. Since $\partial \sigma(A) \subseteq \pi_{o o}(A) \cup \sigma_{l}(A)$ [3], we find $0 \in \pi_{o o}(A) \cup \sigma_{l}(A)$ and hence $0 \in w(A) \cup \pi_{o o}(A)$. Again we get the contradiction.

LEMMA 3.18. $w(A) \cap \pi_{o o}(A)=\phi$

Proof. Suppose $z \in \pi_{o o}(A)$. If $\mathrm{z}$ is non-zero, then it will follow from Lemma 3.15 that $z \in \pi_{o o}(T) \backslash\{0\}$. Since $\pi_{o o}(T) \backslash\{0\}$ and $w(T) \backslash\{0\}$ are disjoint and also $w(A) \backslash\{0\}=w(T) \backslash\{0\}$, one can see that $z$ is not in $w(A)$. Next assume that $z=0$. If $0 \in \sigma(A)$, then 0 is an isolated point of $\sigma(T)$ and therefore an eigenvalue of $T$. This contradicts the injectivity of $T$; thus 0 does not belong to $\sigma(T)$ or $T$ is unitary.

Now we show that $N\left(A^{*}\right)$ is finite dimensional. Note that $N(A)=$ $\left\{(x, y): x \in \overline{R(A)}, y \in N\left(A^{*}\right)\right.$ and $\left.T x+S y=0\right\}$. Let $y_{1}, y_{2}, \ldots, y_{k}$ be linearly independent vectors in $N\left(A^{*}\right)$. Since $T$ is unitary and $S y_{i} \in \overline{R(A)}$, there exist vectors $x_{1}, x_{2}, \ldots, x_{k}$ in $\overline{R(A)}$ such that $T x_{i}+S y_{i}=0$ for $i=1,2, \ldots, k$. Clearly $\left(x_{i}, y_{i}\right) \in N(A)$. One can easily check the linear independence of vectors $\left(x_{1}, y_{1}\right), \ldots,\left(x_{k}, y_{k}\right)$. Thus we have shown that $\operatorname{dim} N\left(A^{*}\right) \leq \operatorname{dim} N(A)$. Since $0 \in \pi_{o o}(A), N\left(A^{*}\right)$ is finite dimensional. We now complete the proof by proving $0 \notin w(A)$. Write

$$
A=\left[\begin{array}{ll}
T & 0 \\
0 & I
\end{array}\right]+\left[\begin{array}{ll}
0 & S \\
0 & -I
\end{array}\right]
$$

Since $N\left(A^{*}\right)$ is of finite dimension, the operator

$$
\left[\begin{array}{ll}
0 & S \\
0 & -I
\end{array}\right]
$$

is compact. As $A$ is the sum of a unitary operator and the compact operator, we conclude that $A$ is a Fredholm operator of index 0 or equivalently, $0 \notin w(A)$ $[1]$.

THEOREM 3.19. A quasi-isometry satisfies Weyl's theorem.

Proof. The result follows from Lemma 3.16, Lemma 3.17 and Lemma 3.18 .

Corollary 3.20. If $A$ is compact, then

(i) $A$ is finite dimensional.

(ii) $N(A)$ is infinite dimensional.

Proof. Under the hypothesis, $T$ is compact. But $T$ being an isometry, its range must be closed and therefore it must be finite dimensional. Since the domain of $T$ is $\overline{R(A)}$ and $T$ is injective, we conclude that $\operatorname{dim} R(A)<\infty$. This proves (i). To prove (ii), note that $T$ is unitary by the first part of the 
proof. The fact that $w(A)=\{0\}$ and Theorem 3.19 gives that $0 \notin \pi_{o o}(A)$. This verifies (ii).

\section{General Remarks}

It is well known that the eigenspaces of an isometry are reducing subspaces. However, the corresponding result for a quasi-isometry is invalid. To see this, consider the operator

$$
A=\left[\begin{array}{ll}
T & S \\
0 & 0
\end{array}\right]
$$

on $H \oplus H$, where $T$ is an isometry with non-empty point spectrum and $S$ is invertible. Observe that $\pi_{o}(A) \neq \phi$. We claim that for each $\lambda \in \pi_{o}(A)$, $N(A-\lambda I) \nsubseteq N\left(A^{*}-\lambda^{*} I\right)$. Suppose $(A-\lambda I)(x, y)=0$ for some $(x, y) \neq 0$. Then $(T-\lambda I) x+S y=0$ and $-\lambda y=0$. If $(x, y) \in N\left(A^{*}-\lambda^{*} I\right)$, then $\left(T^{*}-\lambda^{*} I\right) x=0$ and $S^{*} x-\lambda^{*} y=0$. Since $\lambda y=0$, the first equation gives $S^{*} x=0$ and so the invertibility of $S$ forces $x=0$. Consequently the equation $(T-\lambda I) x+S y=0$ yields $S y=0$ or $y=0$. Thus we have $(x, y)=0$, which is a contradiction. Therefore for no $\lambda \in \pi_{o}(A), N(A-\lambda I) \subseteq N\left(A^{*}-\lambda^{*} I\right)$.

In [6, Remark to Theorem 4], we have raised the following question: is it true that a quasi-isometry is normal if it is reduced by its null space? The answer turns out to be no as can be seen by the following counter example.

Example 4.1. Let $T$ be the unilateral shift on $H$. Define an operator $S$ on $H$ by $S x=0$ if $x \in R(T)$ and $S x=2 x$ if $x \in N\left(T^{*}\right)$. Then $S$ is self-adjoint and $N(S)=R(T)$. Let

$$
A=\left[\begin{array}{ll}
T & S \\
0 & 0
\end{array}\right] .
$$

Then $A$ is a quasi-isometry on $H \oplus H$. We first show that $N(A) \subseteq N\left(A^{*}\right)$. Suppose $A(x, y)=0$. Then $T x+S y=0$. Since $N(S)=R(T)$, we find $S y \in N(S)$. But then $S^{2} y=0$ and hence $S y=0$ as $S$ is selfadjoint. This together with $T x+S y=0$ will imply $x=0$. Consequently $A^{*}(x, y)=0$. Thus $N(A) \subseteq N\left(A^{*}\right)$. The construction of $S$ shows that $\|S\| \geq 2$ and $S T=0$. In view of Theorem 3.3, $A$ is non-hyponormal.

If $A$ is a quasi-isometry, then $|W(A)| \geq 1$ because $r(A)=1$. In case $|W(A)|=1$, it is obvious that $A$ is spectraloid, i.e., $r(A)=|W(A)|$. Here the possibility for a stronger conclusion is not known to us. However, if $A$ is idempotent, then it turns out be a projection [4].

ACKNowledgements.

The author is thankful to the referee for his comments and suggestions over the original version of the article. 


\section{REFERENCES}

[1] S.K. Berberian, The Weyl spectrum of an operator, Indiana Univ. Math. J. 20 (1970), $529-544$.

[2] L.A. Coburn, Weyl's theorem for non-normal operators, Michigan Math. J. 13 (1965), 285-288.

[3] P.A. Filmore, J.G. Stampfli and J.P. Williams, On the essential numerical range, the essential spectrum and the problem of Halmos, Acta Sci. Math. (Szeged) 33 (1972), 19-192.

[4] T. Furuta and Nakamoto, Certain numerical radius contraction operators, Proc. Amer. Math. Soc. 29 (1971), 521-524.

[5] P.R. Halmos, A Hilbert Space Problem Book, Springer Verlag, New York, 1978.

[6] S.M. Patel, A note on quasi-isometries, Glasnik Matematički 35(55) (2000), 113118.

[7] J.G. Stampfli, Hyponormal operators, Pacific J. Math. 12 (1962), 1453-1458.

[8] J.G. Stampfli, Hyponormal operators and spectral density, Trans. Amer. Math. Soc. 117 (1965), 469-476.

S.M. Patel

Department of Mathematics

Sardar Patel University

Vallabh Vidyanagar, Gujarat

India

Received: 01.06.2001.

Revised: 08.11.2002. 